Marquette University

e-Publications@Marquette

Biological Sciences Faculty Research and

Publications

Biological Sciences, Department of

2-1-1966

Transposition and Replication of Modulator in Maize

Irwin M. Greenblatt

Marquette University

Accepted version. Genetics, Vol. 53, No. 2 (February 1966): 361-369. Permalink. (C) 1996 Genetics Society of America. Used with permission. 


\title{
TRANSPOSITION AND REPLICATION OF MODULATOR IN MAIZE ${ }^{1}$
}

\author{
IRWIN M. GREENBLATT \\ Department of Biology, Marquette University \\ Milwaukee, WI
}

PRIOR to the discovery of transposable elements it would have seemed absurd to discuss a chromosomal specificity other than in the context of a fixed position on a chromosome (barring gross rearrangement). It is now well recognized that a chromosomal specificity may indeed not enjoy a fixed locus in a linear array (MCCLINTOCK 1950, 1951, 1956; BRINK et al. 1952; BRINK 1954; PETERSON 1961). It would also seem axiomatic to depict the replication cycle of a chromosomal specificity as the same as that of the entire chromosome, but again, a transposable element seems to be able to replicate more often than the chromosome on which it resides (GREENBLATT and BRINK 1962, 1963). What follows, provides additional evidence in support of an hypothesis that a transposable element, specifically, Modulator, may replicate twice, by reason of transposition, while the chromosome on which it resides replicates once during a single mitosis.

\footnotetext{
${ }^{1}$ Supported by grants G17868 and GB-3576 from the National Science Foundation.

Genetics 53: 361-369 February 1966.
}

Genetics, Vol. 53, No. 2 (February 1966): pg. 361-369. DOI. This article is @ Genetics Society of America and permission has been granted for this version to appear in e-Publications@Marquette. Genetics Society of America does not grant permission for this article to be further copied/distributed or hosted elsewhere without the express permission from Genetics Society of America. 
Modulator $\left(M_{p}\right)$, when conjoined with the Prr allele (red pericarp and cob) suppresses the pigment-producing potential of this allele. $M_{p}$ may transpose away from the Prr locus to one or another site, usually on the same chromosome, resulting in full expression of the fixed chromosomal gene. Since transposition occurs only in some cell lineages, the resultant phenotype is a variegation of red and colorless areas in the pericarp tissue. A single $M_{p}$ element, when initially present in a variegated plant at the $P$ locus, results in a degree of variegation known as medium variegated $\left(\overline{P^{r r} M_{p}}\right)$. An effect of a transposed Modulator $\left(t r-M_{p}\right)$ in a plant also carrying a $\overline{P^{r r} M_{p}}$ complex is to reduce markedly the frequency with which the $M_{p}$ at the $P$ locus transposes. The ear phenotype is thus altered to one with few red stripes known as light variegated $\left(\overline{P^{r r} M_{p}}+t r-M_{p}\right)$. The red or light variegated phenotype may occur over the whole of an ear in the progeny of a medium variegated parent. These two "mutant" phenotypes may also occur as sectors on otherwise medium variegated ears-often as recognizable and well defined sectors of red twinned with light variegated.

It was originally believed that the $M_{p}$ element lacking from the $P$ locus in a twinned red sector was the $t r-M_{p}$ element found in the contiguous light variegated tissue (BRINK and NILAN 1952). Further genetic analysis of such twinned red-light variegated sectors (GREENBLATT and BRINK 1962; GREENBLATT, unpublished) led to the following observations and conclusions:

1. Two types of twin mutations occur with respect to the presence or absence of a $t r-M_{p}$ element in the red co-twin. While phenotypically indistinguishable on the basis of pericarp color, $62 \%$ of the red sectors analyzed contained a tr-Mp element somewhere in their genome while $38 \%$ were totally void of a Modulator anywhere in their genome (light variegated regularly carries a tr-Mp).

2. The tr-Mp element when present in the red co-twin was mapped to the same chromosomal site as the tr-Mp found in its lightvariegated co-twin (twins of independent origin yield detectably different sites).

3. In order to account for the class of twin mutations which carry a tr-Mp in their red co-twin, it was postulated that they result 
from a single transposition of $M p$ during the time of mitotic chromosome replication. $P^{r r}$ and its conjoined $M p$ are assumed to replicate at the $P$ locus, producing two $\overline{P^{r r} M_{p}}$ complexes, prior to replication of certain other portions of the chromosome. An $M p$ from one of the two newly formed daughter PrrMp complexes transposes to an as yet unreplicated site, and then replicates a second time in phase with the chromosome in that region.

Completion of the mitotic cycle results in daughter nuclei of two genotypes: (a) $P^{r r}+t r-M p$, conditioning red pericarp, and (b) $\overline{P^{r r} M_{p}}+t r-M p$, which gives rise to the light variegated phenotype.

Since the seed used from all the twin sectors analysed represent a highly selected sample of gametes from a medium variegated parent, it is therefore of major importance to determine if a random selection of gametes from such a parent yields the same results. In addition, the twin spots analysed resulted from transpositional events occurring early in the ontogeny of the pericarp while the majority of the transpositions of $M p$ away from the $P$ locus occur late in development (WOOD and BRINK 1956). Do these more frequent late transpositions behave in the same manner as in twin spot formation? An analysis for the presence of $t r-M p$ was therefore made of a random sample of offspring reared from a medium variegated parent in order to ascertain the frequency of transposition with respect to the frequency of $t r-M p$ replication.

\section{Materials and Methods}

The individuals serving as test material to be scored for the presence or absence of a $t r-M p$ element all arose from the mating of heterozygous medium variegated pericarp plants (all of a single sibship) serving as female parents and colorless pericarp red cobbed $\left(P^{w r}\right)$ individuals (also from a single sibship) serving as pollen parents. These two parent types are presumed to differ only at the pericarp locus; i.e. the medium variegated parent being $\overline{P^{r r} M_{p}} / P^{w r}$ while the colorless pericarp parent is $P^{w r} / P^{w r}$. The variegated type had previously undergone eight consecutive backcross matings to the male type (inbred W23).

Genetics, Vol. 53, No. 2 (February 1966): pg. 361-369. DOI. This article is @ Genetics Society of America and permission has been granted for this version to appear in e-Publications@Marquette. Genetics Society of America does not grant permission for this article to be further copied/distributed or hosted elsewhere without the express permission from Genetics Society of America. 
Seed used to rear plants for tr-Mp evaluation were selected only from ears judged to be medium variegated, Confirmation of correct judgment is afforded by the distribution of the different variegated types reared from these seed. All seed available from the parent medium variegated ears were planted, with a single important exception. If an ear contained somatic sectors that were not medium variegated (primarily red and light variegated spots) overlying more than one kernel only a single randomly chosen kernel from each such somatic sector was included in the random sample of seed. The remaining seed from these sectors were discarded. Such seed selection assures that no multiple chromosome copies from a single transpositional event are included in the tested progeny and that each "mutant" arising in the tested progeny is of independent origin.

Each of the plants reared from the selected medium variegated seed was tested for the presence or absence of a tr-Mp element. Since a transposable element does not condition a visible autonomous phenotype, the detection of tr-Mp was accomplished by pollinating each of the plants with a specific tester line. This tester stock, used as a male in a detasseling plot design, carried, in addition to the dominant, complementary aleurone color genes, $A, C$, and $R$, the Dissociation (Ds) factor in the standard position proximal to the $C$ locus on the short arm of chromosome 9. Ds, in the form here used, promotes chromosome breakage at its locus only if either Activator (MCCLINTOCK 1951) or Modulator, its counterpart in variegated pericarp strains (BARCLAY and BRINK 1954), is present in the genome. Breakage at $D s$ leads to the loss of the dominant $C$ factor, thus producing a distinctive colored-- colorless mottling pattern on the tested ears since the variegated line is homozygous $C$.

By using the $D s$ tester as a test system each plant to be evaluated, in effect, is in turn progeny tested for the segregation of the $t r-M p$ factor. Thus $t r-M p$ is seen as the occurrence of a $1: 1$ ratio of kernels with and without the $C \rightarrow c$ breakage pattern on the ears tested. In scoring the variegated pericarp class of plants, however, the $C \rightarrow c$ mottling was not used to determine the presence of $t r-M p$, but rather the grade of pericarp variegation of each individual was recorded. Defense of this method has already been given (GREENBLATT and BRINK 1962).

Genetics, Vol. 53, No. 2 (February 1966): pg. 361-369. DOI. This article is @ Genetics Society of America and permission has been granted for this version to appear in e-Publications@Marquette. Genetics Society of America does not grant permission for this article to be further copied/distributed or hosted elsewhere without the express permission from Genetics Society of America. 
In scoring for $\operatorname{tr}-M p$ by the means employed the detected transpositions could have occurred during two different stages of the life cycle of the tested plants. A portion of the total transpositions occurred late in the generation of the medium variegated parent. In these cases meiosis would have intervened between the time of transposition and detection at the end of the following generation. Thus the distribution of a tr-Mp element in the test plants is affected by meiotic recombination. That $P^{w r}$ segregants (see results) are found containing a $t r-M p$ is direct proof that recombination of $t r-M p$ does indeed occur. In addition transpositions can occur from the time meiosis is complete until the test plant forms pericarp tissue relatively late in the development of the plant. In such cases distribution of $\operatorname{tr}-M p$ is governed by a mitotic segregation. The extent of both meiotic and mitotic recombination of $t r-M p$ is dependent upon where the receptor site is in the genome with respect to the $P$ locus. In both cases, however, neither event will in any way influence the number of tr-Mp's detectable, but rather, in which phenotypic class they would he uncovered.

\section{RESULTS}

Expected distribution of tr-Mp: The significance of the number of Modulator elements recoverable after transposition from the $P$ locus is dependent upon recognition of the following considerations: (1) the parental chromosome (medium variegated) contains one $\mathrm{P}^{\mathrm{rr}}$ allele and one $M p$ element. (2) Each red offspring among the progeny represents an independent transposition of $M p$ away from the $P$ locus. (3) A tr-Mp element detected in a plant of the test generation, at any site other than the $P$ locus, represents a recovery of the element after transposition; this is true for all phenotypic classes. The problem is then reduced to one of totaling the number of transpositions and the number of $t r-M p$ elements among the offspring of a medium variegated parent. A listing of these expectations with respect to phenotypic classes found is presented in Table 1.

Since only those Modulator elements not at the $P$ locus are recordable as having transposed, the parental classes of medium variegated and colorless pericarp would not contribute to a final tally of Modulator elements recoverable. In the cases of the "mutant" class 
NOT THE PUBLISHED VERSION; this is the author's final, peer-reviewed manuscript. The published version may be accessed by following the link in the citation at the bottom of the page.

both the red and colorless pericarp containing a tr-Mp $(M p+)$ are phenotypically indistinguishable from those plants void of the element

Genetics, Vol. 53, No. 2 (February 1966): pg. 361-369. DOI. This article is @ Genetics Society of America and permission has been granted for this version to appear in e-Publications@Marquette. Genetics Society of America does not grant permission for this article to be further copied/distributed or hosted elsewhere without the express permission from Genetics Society of America. 
NOT THE PUBLISHED VERSION; this is the author's final, peer-reviewed manuscript. The published version may be accessed by following the link in the citation at the bottom of the page.

TABLE 1

A reference listing of the number of Modulator elements present in each of the phenotypic classes under discussion

\begin{tabular}{ccccc}
\hline & Pericarp type & & \multicolumn{2}{c}{ Number of modulators } \\
\cline { 3 - 4 } & "Mutant" & & At $P$ locus & Transposed \\
\hline medium variegated & & 1 & 0 \\
$p^{\text {vor }}\left(M p^{-}\right)$ & red $\left(M p^{+}\right)$ & 0 & 0 \\
& red $\left(M p^{-}\right)$ & 0 & 1 \\
& $p^{\text {vor }}\left(M p^{+}\right)$ & 0 & 0 \\
& light variegated & 0 & 1 \\
& very light variegated & 1 & 1 \\
& & 1 & $1^{*}$ \\
\hline
\end{tabular}

* Scored as one-two may be present. See text

$\left(M p^{-}\right)$. Detection of $\operatorname{tr}-M p$ is by means of the $D s$ tester. With reference to the variegated "mutants," both light and very light, only a single tr$M p$ is postulated. The rationale used to judge a very light variegated as carrying only a single tr-Mp when the phenotype would seem to indicate that two are present is governed by the probability (presumably $p=1 / 2$ ) that it was the first $\operatorname{tr}-M p$ which gave rise to the second by means of a secondary transposition. In which case it should not enter into a tally of $t r-M p$ 's emanating from the $P$ locus directly. The transpositional event, if considered solely as a displacing event in the history of a Modulator element, should in no way affect the number of these elements with respect to the number of $P^{\text {rr }}$ alleles present in a sample. Finding fewer tr-Mp elements than the number of transpositions would indicate either a biological loss at the time of transposition or inefficient recovery. Finding an excess of $t r-M p$ elements over the number of transpositions, in spite of recovery loss, would mean that the element can replicate more often than the chromosome as a whole.

Observed distribution of tr-Mp: The distribution of phenotypic classes arising from the test mating $\overline{P^{r r} M_{p}} / P^{w r} \times P^{w r} / P^{w r}$ is listed in Table 2 . The proportion of colored to colorless offspring is equal,

Genetics, Vol. 53, No. 2 (February 1966): pg. 361-369. DOI. This article is @ Genetics Society of America and permission has been granted for this version to appear in e-Publications@Marquette. Genetics Society of America does not grant permission for this article to be further copied/distributed or hosted elsewhere without the express permission from Genetics Society of America. 
NOT THE PUBLISHED VERSION; this is the author's final, peer-reviewed manuscript. The published version may be accessed by following the link in the citation at the bottom of the page.

\section{TABLE 2}

Segregation of pericarp phenotypes among the offspring arising from the mating: $\overline{\operatorname{Pr} M p} / \mathrm{Pwr} \times \mathrm{Pwr} / \mathrm{Pwr}$

\begin{tabular}{|c|c|c|c|}
\hline \multicolumn{2}{|c|}{ Pericarp phenotype } & \multirow{2}{*}{$\begin{array}{l}\text { Number of } \\
\text { individuals }\end{array}$} & \multirow[b]{2}{*}{ Percent } \\
\hline Colored & Colorless & & \\
\hline \multicolumn{2}{|l|}{ medium variegated } & 2,301 & 42,10 \\
\hline \multicolumn{2}{|l|}{ red } & 283 & 5.18 \\
\hline \multicolumn{2}{|l|}{ light variegated } & 160 & 2.93 \\
\hline \multicolumn{2}{|l|}{ very light variegated } & 14 & 0.25 \\
\hline \multicolumn{2}{|l|}{ orange variegated } & 1 & 0.02 \\
\hline \multirow[t]{3}{*}{ Total } & & 2,759 & 50.48 \\
\hline & Pwor/Pwr & 2,707 & 49.52 \\
\hline & & 5,466 & \\
\hline
\end{tabular}

indicating that the two chromosome one homologs are segregating in a normal manner. It also indicates that neither chromosome breakage nor distortion of a normal meiotic disjunction is caused by the presence of the transposable element. Within the colored class of offspring the expected distribution of red, light variegated, and very light variegated ears, for the W23 inbred background, is realized (BRAWN 1956). The single orange variegated ear is also expected (BRAWN 1956), but it is not as yet clear what type of transpositional event is involved. Since orange variegated is a single item among a total of 5,466 it is not further considered in the analysis of $M p$ transposition and replication.

Based on the number of red ears (283 total) within the colored class $(2,759$ total) the frequency of $M p$ transposition from the $P$ locus is calculated to be $10.2 \%$. Thus $89.9 \%$ of the recovered $P^{r r}$ containing chromosomes still have the original $M p$ conjoined at the $P$ locus. The colored class also includes the light and very light variegated types which contain, in addition to the $M p$ at the $P$ locus, a tr-Mp elsewhere in the genome (locations unknown). 
Where and with what frequency tr-Mp is found among all the classes of offspring can not be determined from these data alone. Table 3 presents the necessary data relating to tr-Mp distribution and frequency among all the offspring. From the table it is seen that 89 colorless ears from a total of 2,707 were found to carry a tr-Mp element somewhere in their genome. By the same detection techniques tr-Mp was found among the red segregants in 145 cases and the element was totally absent in the remaining 138 cases. The total of recovered $t r-M p$ elements in this population of test ears is 408 . Within this same population of ears the number of transpositions of $M p$ from the $P$ locus (total number of red ears) is found to be only 283. Using these data to compute the ratio of detected $\operatorname{tr}-M p$ elements per transposition the ratio is found to be $1.44 \mathrm{tr}-\mathrm{Mp}: 1.0$ transposition. A $x^{2}$ analysis of goodness of fit of these data to an expectation of $1: 1$ (one transposition for one $\operatorname{tr}-M p$ element) yields a $P$ value of $<.001$. Thus more tr-Mp elements are recovered than can be accounted for solely on the basis of movement of $M p$ from the $P$ locus to other sites on the chromosomes.

Recoverability of tr-Mp: As already mentioned, the two chromosome-1 homologs in the test generation are being recovered

\section{TABLE 3}

\section{Distribution and frequency of transposed Modulator and the $\mathrm{Prr}$ allele (not conjoined) in the offspring arising from the mating $\overline{\mathrm{PrrMp}} / \mathrm{Pwr} \times \mathrm{Pwr} / \mathrm{Pwr}$}

\begin{tabular}{lcc}
\hline Pericarp phenotype & Number of transposed Mp & Number of transpositions \\
\hline medium variegated & 0 & 0 \\
por $\left(M p^{\dagger}\right)$ & 89 & 0 \\
red & 145 & 283 \\
light variegated & 160 & 0 \\
very light variegated & 14 & 0 \\
& & 283 \\
Totals & 408 & 28 \\
\hline
\end{tabular}

Genetics, Vol. 53, No. 2 (February 1966): pg. 361-369. DOI. This article is @ Genetics Society of America and permission has been granted for this version to appear in e-Publications@Marquette. Genetics Society of America does not grant permission for this article to be further copied/distributed or hosted elsewhere without the express permission from Genetics Society of America. 
NOT THE PUBLISHED VERSION; this is the author's final, peer-reviewed manuscript. The published version may be accessed by following the link in the citation at the bottom of the page.

with equal frequency as expected. But what the data do not show is the absolute recovery rate of a tr-Mp element after transposition. The data in Table 3 yield only relative values of tr-Mp recovery with respect to the number of postulated transpositions. In order to gain absolute values of $t r-M p$ recovery frequency a series of red sectors co-twinned to light variegated sectors were tested for the presence or absence of tr-Mp. In such red multikernel sectors a tr-Mp would only be present if a second replication of $t r-M p$ had occurred at the time of transposition from the $P$ locus. Scoring the segregation of $t r-M p$ in the red sector serves as a test of $t r-M p$ recovery after the transposition. In addition, in these twinned red sectors, the sequence of events (i.e. transposition and replication) is totally definable and has occurred prior to the transmission of the element to the test generation (GREENBLATT and BRINK 1962). The results of testing 31 multikernel red co-twin sectors for $\operatorname{tr}-\mathrm{Mp}$ recoverability are presented in Table 4 . From the totals at the bottom of the table it can be seen that both the red and colorless pericarp classes carry the tr-Mp element in the test generation. Of the total of 296 test individuals, 130 are found to contain a tr-Mp while 166 are found to be completely void of the element. Since only a single element is postulated in these sectors after transposition, a ratio of one $M p^{+}$: one $M p^{-}$is expected. A $x^{2}$ test of goodness of fit for this expectation yields $P=.05-.02$. Thus recovery of $t r-M p$ after transposition is low in efficiency but not highly significantly so. Such loss of detectable tr-Mp elements would seem to minimize the ratio of tr-Mp replications per transposition.

\section{DISCUSSION}

That each red offspring is indeed the result of a transposition of $M p$ away from the $P$ locus is basic to the thesis under discussion. Defense of this proposition comes from the following considerations: Approximately $49 \%$ of a total of 283 independently occurring red pericarp ears are found, by the $D s$ test, to be completely void of an $M p$ element. In these cases Modulator has not only transposed from the $P$ locus but has become affixed to a chromosomal site sufficiently distant from the $P$ locus to insure its segregation from $P$ either during the mitosis involved or a subsequent meiosis. The location of $\operatorname{tr}-M p$ in the genome of the remaining half of red ears in the present sample is

Genetics, Vol. 53, No. 2 (February 1966): pg. 361-369. DOI. This article is @ Genetics Society of America and permission has been granted for this version to appear in e-Publications@Marquette. Genetics Society of America does not grant permission for this article to be further copied/distributed or hosted elsewhere without the express permission from Genetics Society of America. 
unknown. However, when linkage analyses were made of $t r-M p$ in another sample of 32 independent large red somatic sectors (GREENBLATT and BRINK 1962; GREENBLATT unpublished) in which a tr-Mp was present, tr-Mp was found to recombine with the $P$ locus in all cases but one. That single exception, as with the other somatic red sectors, was a co-twin to an adjacent light variegated sector indicating that a second replication had occurred and that $t r-M p$ had transposed away from the $P$ locus, but apparently too short a distance from the $P$ locus for measurement. Phenotypically this red sector was indistinguishable from all others studied. MCCLINTOCK(1956), in a series of similar cases of Activator $(A C)$ conjoined at the Bronze $(B z)$ locus on chromosome 9, examined 14 independent "mutations" to full $B z$ expression. She found that each represented the removal of $A C$ from the $B z$ locus, as with $M p$ at the $P$ locus.

Genetics, Vol. 53, No. 2 (February 1966): pg. 361-369. DOI. This article is @ Genetics Society of America and permission has been granted for this version to appear in e-Publications@Marquette. Genetics Society of America does not grant permission for this article to be further copied/distributed or hosted elsewhere without the express permission from Genetics Society of America. 
NOT THE PUBLISHED VERSION; this is the author's final, peer-reviewed manuscript. The published version may be accessed by following the link in the citation at the bottom of the page.

\section{TABLE 4}

Frequency of tr-Mp among the progeny reared from multikernel red sectors (co-twins with light variegated) arising on otherwise medium variegated ears.

$$
\text { Mating: } \overline{\mathrm{Prr}^{\mathrm{r}} \mathrm{Mp}} / \mathrm{P}^{\mathrm{wr}} \times \mathrm{P}^{\mathrm{wr}} / \mathrm{P}^{\mathrm{wr}}
$$

\begin{tabular}{|c|c|c|c|c|}
\hline \multirow[b]{2}{*}{ Sector number } & \multicolumn{2}{|c|}{ Red pericarp } & \multicolumn{2}{|c|}{ Colorless pericarp } \\
\hline & Present & ${ }^{t r-M p} \quad$ Absent & Present & ${ }^{t r-M p}$ Absent \\
\hline $64 \mathrm{D}-103$ & 8 & 0 & 2 & 8 \\
\hline-104 & 6 & 3 & 1 & 5 \\
\hline-105 & 0 & 4 & 1 & 7 \\
\hline-106 & & * & . & * \\
\hline-107 & 6 & 2 & 0 & 6 \\
\hline-108 & 1 & 5 & 4 & 6 \\
\hline-109 & 3 & 0 & 0 & 4 \\
\hline-110 & 4 & 6 & 5 & 1 \\
\hline-111 & 5 & 1 & 3 & 4 \\
\hline-112 & 5 & 5 & 3 & 7 \\
\hline-113 & 1 & 2 & 2 & 2 \\
\hline-114 & 6 & 4 & 2 & 8 \\
\hline-115 & & * & & $*$ \\
\hline-116 & & * & . & $*$ \\
\hline-117 & & * & . & $*$ \\
\hline-118 & 1 & 7 & 0 & 5 \\
\hline-119 & 4 & 5 & 5 & 4 \\
\hline-120 & 3 & 6 & 6 & 2 \\
\hline-121 & 7 & 3 & 1 & 5 \\
\hline-122 & 4 & 4 & 3 & 5 \\
\hline-123 & & $*$ & . & $*$ \\
\hline-124 & & $\cdot$ & & $*$ \\
\hline-125 & 6 & 0 & 1 & 3 \\
\hline-126 & & $*$ & & $*$ \\
\hline-127 & 5 & 1 & 1 & 6 \\
\hline-128 & & $*$ & 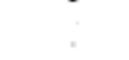 & $*$ \\
\hline-129 & 6 & 5 & 1 & 4 \\
\hline-130 & . & $\cdot$ & . & $*$ \\
\hline-131 & & * & & $*$ \\
\hline-133 & 2 & 4 & 2 & 5 \\
\hline-136 & 4 & 0 & 0 & 2 \\
\hline Totals & 87 & 67 & 43 & 99 \\
\hline
\end{tabular}

- No $t r-M p$ present-tr-Mp presumed not to have replicated second time.

Possible errors that might bias the results toward an increased recovery of tr-Mp are: (1) an extraneous source of $M p$ by unknown contaminating pollen. In the tests reported here this was ruled out by

Genetics, Vol. 53, No. 2 (February 1966): pg. 361-369. DOI. This article is @ Genetics Society of America and permission has been granted for this version to appear in e-Publications@Marquette. Genetics Society of America does not grant permission for this article to be further copied/distributed or hosted elsewhere without the express permission from Genetics Society of America. 
not having any source of such pollen in the field. The medium variegated plants were themselves detasseled prior to pollen shed. (2) Supply to the test generation of multiple copies of the same transpositional event is also excluded owing to the seed selection procedure followed. It is therefore certain that all $t r-M p$ elements recovered in the test generation come from the designated test material.

In fact, the recovered number of tr-Mp elements is believed to represent a minimum. By the means employed to detect $t r-M p$, if two were present in a single plant, and had arisen by independent transpositions, they would be scored as a single representative or, as discussed by (GREENBLATT and BRINK (1962), both might go completely undetected. Another minimizing factor affecting tr-Mp recovery is poor viability of an individual carrying a $t r-M p$ at an unknown site. No data for Modulator are as yet available on this question but MCCLINTOCK (1951) has detected just such dominant lethal effects for $A c$ in some cases of transposition.

It appears that all transpositions do not result in a second replication of the element. Such a conclusion is based on two counts. First, if this were so the recovery frequency of tr-Mp per transposition would be $2: 1$, barring recovery losses. A $x^{2}$ comparison of the observed number of tr-Mp elements per transposition with an expectation of 2 tr-Mp: 1 transposition yields $P<, 001$. Second, and most important, the data obtained from the analysis of 261 red sectors which occurred as co-twins to light variegated sectors (GREENBLATT unpublished data) show that $62 \%$ of these contain a $t r-M p$ while the remainder are totally void. The presence of a $\operatorname{tr}-M p$ in such sectors indicates that a second replication of the element took place at the time of transposition. Why $62 \%$ of the transpositions result in an extra copy of tr-Mp is believed related to the state the receptor site is in with respect to degree of completion of replication. The earlier the stage of replication of the receptor site the greater the probability of $t r-M p$ being again replicated. In each of the twin mutations Modulator is depicted as first replicating at the $P$ locus during chromosome replication, and then one of the replicated $M p$ elements transposes to a receptor site. What happens at the receptor site is the point in discussion.

Genetics, Vol. 53, No. 2 (February 1966): pg. 361-369. DOI. This article is (C Genetics Society of America and permission has been granted for this version to appear in e-Publications@Marquette. Genetics Society of America does not grant permission for this article to be further copied/distributed or hosted elsewhere without the express permission from Genetics Society of America. 
Strikingly, if the results from twin-mutation data are used to set the expectations from a random sample of transpositions, namely 1.62 tr-Mp's per transposition, the data presented here (Table 3) agree with this expectation. That is, the actual recovery of $1.44 \mathrm{tr}-\mathrm{Mp}$ elements per transposition in the present sample is statistically not different from the $1.62: 1.0$ expectation $(P=.25-.1)$.

The two points at issue are: (1) whether twin mutations arise by a different sequence of transposition events from a random sample of such events; and (2) whether there is a detectable change in the mechanism of transposition within different developmental stages of the ear. The data herein reported establish that twin-mutation analysis and the random-sample analysis give indistinguishable results. This suggests that the transposition mechanism is a constant one throughout development, and that all transpositions lead to genetic twin formation.

\section{SUMMARY}

When the total recovered number of transpositions (red ears) is compared with the total number of transposed modulators (tr-Mp) in a random sample of offspring reared from a heterozygous medium variegated parent, an excess of tr-Mp is found. For each transposition event $1.44 t r-M p$ 's are recovered. The basis for the excess of $t r-M p$ is believed to be $M p$ replication at the $\boldsymbol{P}$ locus prior to transposition and a second replication of $M p$ at the receptor site during the same mitotic replication. The frequency of these second replications, while not directly measured in the data presented, fits well the $62 \%$ rate predicted on the basis of earlier studies of twin mutations.

\section{Literature cited}

BARCLAY, P. C., and R. A. BRINK, (1954), The relation between Modulator and Activator in maize. Proc. Natl. Acad. Sci. U.S. 40: 1118-1126.

BRAWN, R. I., (1956), Effect of the $P^{v v}$ allele in homozygous and heterozygous condition on mutations to red and light variegated pericarp in maize. Unpublished Ph.D. thesis. University of Wisconsin Library, Madison.

Genetics, Vol. 53, No. 2 (February 1966): pg. 361-369. DOI. This article is @ Genetics Society of America and permission has been granted for this version to appear in e-Publications@Marquette. Genetics Society of America does not grant permission for this article to be further copied/distributed or hosted elsewhere without the express permission from Genetics Society of America. 
NOT THE PUBLISHED VERSION; this is the author's final, peer-reviewed manuscript. The published version may be accessed by following the link in the citation at the bottom of the page.

BRINK, R. A., (1954), Very light variegated pericarp in maize. Genetics 39: 724-740.

BRINK, R. A., and R. A. NILAN, (1952), The relation between light variegated and medium variegated pericarp in maize. Genetics 37: 519-544.

GREENBLATIT. ,M ., and R. A. BRINK, (1962), Twin mutations in medium variegated pericarp maize. Genetics 47: 489-501. - (1963), Transpositions of Modulator in maize into divided and undivided chromosome segments. Nature $197:$ 412-413.

MCCLINTOCK, B., (1950), The origin and behavior of mutable loci in maize. Proc. Natl. Acad. Sci. U.S. 36: 344-355. - (1951), Chromosome organization and genic expression. Cold Spring Harbor Symp. Quant. Biol. 16: 13-47. - (1956), Controlling elements and the gene. Cold Spring Harbor Symp. Quant. Biol. 21 : 196-216.

PETERSON., P. A., (1961), Mutable $a_{1}$ of the En system in maize. Genetics 46: 759-771.

WOOD, D. R., and R. A. BRINK, (1956), Frequency of somatic mutation to self color in maize plants homozygous and heterozygous for variegated pericarp. Proc. Natl. Acad. Sci. U.S.

42: 514-519.

Genetics, Vol. 53, No. 2 (February 1966): pg. 361-369. DOI. This article is @ Genetics Society of America and permission has been granted for this version to appear in e-Publications@Marquette. Genetics Society of America does not grant permission for this article to be further copied/distributed or hosted elsewhere without the express permission from Genetics Society of America. 